\title{
UNE MÉTHODE STATISTIQUE : L'ANALYSE PROGRESSIVE
}

\author{
C. MILLIER \\ Station de Biométrie, Centre national de Recherches forestières, 54 - Nancy \\ Institut national de la Recherche agronomique
}

SOMMAIRE

On présente les principes d'une méthode statistique, l'analyse progressive : taille d'échantillon non fixée à l'avance, nécessité d'évaluation précise de risques.

Des applications forestières permettent de dégager l'intérêt, les limites et le champ d'application d'une telle technique : malgré ses imperfections, elle constitue un outil non négligeable et une façon différente de raisonner statistiquement, utile dans l'évaluation des projets d'expérience.

\section{INTRODUCTION}

Etant donnée une population $P$ sur laquelle on mesure un caractère et qui possède vis-à-vis de ce caractère une fonction de répartition $F$, étant données des hypothèses que l'on veut vérifier sur la loi $F$, les tests statistiques classiques procurent un moyen de vérifier ces hypothèses, en tirant de $P$ suivant des modalités à observer, ou plan d'échantillonnage, un échantillon $E$ de taille donnée et en se donnant un risque de se tromper dans le refus ou l'acceptation d'une hypothèse.

L'analyse progressive (en anglais (( sequential analysis )) part d'un autre point de vue : soit à choisir entre deux hypothèses $H_{0}$ et $H_{1}$, peut-on construire un plan d'échantillonnage consistant à tirer un à un des individus de $P$ et à s'arrêter quand on a l'information nécessaire pour choisir entre les deux hypothèses $H_{0}$ et $H_{1}$ ?

La réponse est oui, la méthode que nous allons étudier est en général moins coûteuse en tirages que le plan d'échantillonnage classique ; elle possède néanmoins des particularités qui en restreignent sensiblement l'usage.

Découverte pendant la guerre, cette technique fut, paraît-il, à cause de ce gain de temps donc d'argent (qu'on avait peut-être surestimé au départ) décrétée secret 
militaire. Elle a fait l'objet d'une somme de travaux théoriques très importante depuis 1945 et elle est appliquée très souvent dans le contrôle des fabrications industrielles aux Etats-Unis.

Depuis quelques années, elle a fait son apparition dans les sciences biologiques, tout naturellement; en médecine, il paraît en effet intéressant d'arrêter une expérimentation de médicament (ou autre) dès que l'on ( voit ) statistiquement que l'on s'est trompé.

Nous donnerons plus loin quelques exemples d'application aux sciences agrcnomiques et forestières.

Après une introduction à la notion de puissance, qui est également essentielle dans l'optimisation des plans d'expérience, nous étudierons les principales caractéristiques des tests progressifs et l'application à des problèmes classiques.

Cet article, qui se veut uniquement vulgarisateur d'une technique peu connue est basé sur les travaux de WALD (1947), l'inventeur, de JoHNSON (1961) et de ANDERSON (1960).

\section{1. - PRINCIPE DES TESTS STATISTIQUES}

\section{1. - But des tests statistiques}

Une population possède vis-à-vis d'un caractère déterminé une fonction de répartition $F$. Le but du test statistique en général est de tirer des conclusions sur la validité d'une hypothèse $H$ formulée sur la fonction de répartition $F$, et ce au vu d'une partie seulement de la population, l'échantillon.

Un test peut être défini comme étant l'ensemble des points suivants :

a) une formulation de l'hypothèse $H$ à accepter ou à refuser (ou des hypothèses entre lesquelles il nous faut choisir) ;

b) une règle d'échantillonnage pour le tirage des individus ;

c) une règle de décision : au vu de l'échantillon, décider si l'hypothèse à tester doit être acceptée ou refusée ; c'est-à-dire avoir coupé l'ensemble des échantillons observables en deux, le sous-ensemble de ceux pour lesquels $H$ doit être acceptée et son complémentaire.

Il nous faut maintenant préciser les points $a$ ) et $c$ ).

\section{2. - Types d'hypothèses}

a) On peut facilement s'apercevoir que tester une hypothèse déterminée revient à choisir entre deux hypothèses : par exemple, si nous voulons tester $\left\{H: \mu=\mu_{0}\right\}$ pour une population normale de moyenne $\mu$ inconnue et d'écart-type connu $\sigma$, nous testons en réalité $\left\{H: \mu=\mu_{0}\right\}$ contre l'hypothèse alternative $\left\{H^{\prime}: \mu>\mu_{0}\right.$ ou $\left.\mu<\mu_{0}\right\}$. Le problème se ramène donc toujours au choix entre deux hypothèses.

$b)$ Une hypothèse peut être simple ou composée. La fonction $F$ est définie par plusieurs $(p)$ paramètres ( 2 pour la loi normale, 1 pour la loi binomiale). Une hypothèse sera simple si elle détermine uniquement la valeur des $p$ paramètres, composée 
dans le cas contraire. Ainsi, $\left\{H: \mu=\mu_{0}\right\}$ pour une population normale de moyenne $\mu$ inconnue et d'écart-type connu $\sigma$ est une hypothèse simple alors que c'est une hypothèse composée si $\sigma$ est inconnu $\left\{H: \mu=\mu_{0} ; \sigma=\sigma_{1}\right.$ ou $\sigma_{2}$ ou n'importe quelle valeur\}.

\section{3. - Détermination de la coupure - Notion de risque et de puissance}

Pour exposer cette section, nous nous plaçons dans le cas où $H$ et $H^{\prime}$ sont des hypothèses simples.

En acceptant $H$ aux dépens de $H^{\prime}$ au vu de l'échantillon, nous prenons le risque $(\beta)$ qu'en réalité c'est l'hypothèse $H^{\prime}$ qui prévaut au niveau de la population. De même on prend un autre risque $(\alpha)$ en acceptant $H^{\prime}$ si c'est $H$ qui est vrai.

La coupure en deux de l'espace des échantillons est parfaitement définie si on connaît $\alpha, \beta$; de plus, il existe une relation fonctionnelle entre $n, \alpha, \beta$.

Dans les méthodes d'échantillonnage classiques, on se donne $\alpha$ et $n$; dans les méthcdes d'échantillonnage progressif, $\alpha$ et $\beta$.

Très souvent, les hypothèses à tester sont en quelque sorte hiérarchisées; par exemple, le but de l'analyse de variance est de tester en priorité l'hypothèse nulle $\left\{H: \mu_{1}=\mu_{2}=\ldots=\mu_{k}=\mu\right\}$ c'est-à-dire l'égalité des moyennes de $k$ populations normales : cette hypothèse joue un rôle particulier et prioritaire puisque toute la théorie de l'analyse de variance en découle.

$\alpha$ sera le risque de première espèce pour l'hypothèse nulle (en anglais ( size ») $\operatorname{Pr}\{$ rejeter $H$ si $H$ est vrai $\}=\alpha$

$\beta$ sera appelé risque de deuxième espèce, et $1-\beta$ puissance du test (en anglais (( power ))

$$
\operatorname{Pr}\{\text { accepter } H \text { si } H \text { est faux }\}=\beta(1)
$$

$\alpha$ et $\beta$ sont généralement exprimés en $\%$ ( $c f$. page suivante).

\section{4. - Digression sur la notion de puissance}

L'expérimentateur désire en général obtenir aux moindres frais des renseignements de son plan d'expérience. Une question qu'il se pose souvent est de savoir a priori combien de répétitions mettre en place quand il a une idée sur la valeur des différences qu'il désire mettre en évidence sur l'effet des traitements contrôlés.

Considérons par exemple un simple test $t$ à effectuer sur une population :

$$
\left\{H: \mu=\mu_{0}(\sigma \text { inconnu })\right\}
$$

on définit $H^{\prime}$ l'hypothèse alternative

$$
\left\{H^{\prime}: \mu \leqslant \mu_{0}-\delta \text { ou } \mu \geqslant \mu_{0}+\delta\right\}
$$

où $\delta$ est la différence que l'on veut mettre en évidence. Si on se donne $\alpha$ et $\beta$

$$
\operatorname{Pr}\left\{\text { accepter } H \text { si } H^{\prime} \text { est vrai }\right\}=\beta
$$

(1) $\mathrm{Si}$ on ne considère (pour simplifier) que des lois continues et deux hypothèses $\left[H_{0}: f(x)=f_{0}(x)\right.$ pour $\forall x]$ et $\left[H_{1}: f(x)=f_{1}(x)\right.$ pour $\left.\forall x\right]$ (où $f(x)$ est la densité de probabilité), Neymann et Pearson ont montré que la coupure «la meilleure 》dans l'espace des échantillons $x_{1}, x_{2} \ldots x_{n}$ est telle que : $\frac{f_{1}\left(x_{1}\right) f_{1}\left(x_{2}\right) \ldots f_{1}\left(x_{n}\right)}{f_{0}\left(x_{1}\right) f_{0}\left(x_{2}\right) \ldots f_{0}\left(x_{n}\right)} \geqslant k$ ( $k$ tel que la région soit de « size » pour l'hypothèse $\left.H_{0}\right)$. 
déterminera la valeur de $n$; nous pourrons alors affirmer que notre plan expérimental mettra en évidence des différences de l'ordre voulu dans $100(1-\beta)$ cas (TANG, 1938; SCHEFFE, 1952).

Une méthode fausse dans la théorie est de négliger la notion de puissance : dans ce cas, on se donne la variance $s$ et on fait :

$$
\frac{\sqrt{ } n \delta}{s}>t_{\alpha, n-1}
$$

(où $t_{\alpha, n-1}$ représente la valeur du $t$ de Student au risque $\alpha$ et à $n-1$ degrés de liberté).

Cette méthode sous-estime le nombre $n$, ce qui est un défaut très grave (2).

\section{2. - LE TEST PROGRESSIF — PRINCIPE ET PROPRIÉTÉS}

\section{1. - Principe du test progressif}

Supposons que deux hypothèses $H_{0}$ et $H_{1}$ soient à tester.

a) le nombre d'observations n'est pas fixe,

b) on a préalablement déterminé dans l'espace des échantillons, trois sousensembles mutuellement exclusifs pour chaque valeur du nombre de tirages $m$,

$R_{m}^{0}$ : accepter $H_{0}$,

$R_{m}^{1}:$ accepter $H_{1}$,

$R_{m}$ : continuer, c'est-à-dire procéder à un tirage supplémentaire.

On observe $E:\left\{x_{1}, x_{2}, \ldots x_{n}\right\}$; suivant que $E$ appartient à $R_{m}, R_{m}^{0}$ ou $R_{m}^{1}$ on applique les décisions qui en découlent.

\section{2. - Caractéristiques d'un test progressif}

2.21. La fonction $O C$ (de l'anglais ( operating characteristic ))

Soit $H_{0}$ une hypothèse prédéterminée, et $\theta$ la valeur du (ou des) paramètre (s) de la loi de probabilité; la probabilité d'accepter $H_{0}$ est évidemment une fonction de $\theta, 1$ 'OC :

$$
\begin{aligned}
& \operatorname{Pr}\left\{\text { accepter } H_{0} \text { si } \theta\right\}=L(\theta) \\
& \operatorname{Pr}\left\{\text { refuser } H_{0} \text { si } \theta\right\}=1-L(\theta) .
\end{aligned}
$$

(2) Les résultats sont différents pour la raison suivante : dans le cas du test $t$, 1'équation (1) s'exprime grâce à la densité de probabilité du $t$ de Student non centré (paramètre de décentrage $\delta$ ) alors que (2) s'exprime grâce à la densité de $t$ centré.

Les deux densités de probabilité ne sont pas déductibles l'une de l'autre par translation $\delta$; donc si on choisit $\alpha=\beta$, on ne trouvera pas la même taille d'échantillon. 


\subsection{La fonction $A S N$ (de l'anglais ( average sample number»)}

Dans le test progressif, le nombre de tirages n'est pas fixe, c'est une variable aléatoire qui admet des moments et en particulier une moyenne.

Cette moyenne dépend de la valeur $\theta$ du (ou des) paramètre (s); c'est l'ASN (nombre moyen d'observations à prendre en compte).

\subsection{Influence du test sur l'OC et l'ASN}

a) Soit à tester $\left\{H_{0}: \theta=\theta_{0}\right\}$ contre $\left\{H_{1}: \theta=\theta_{1}\right\}$ on a :

$$
L\left(\theta_{1}\right)=\operatorname{Pr}\left\{\text { accepter } H_{0} \text { si } \theta=\theta_{1}\right\}=\beta
$$

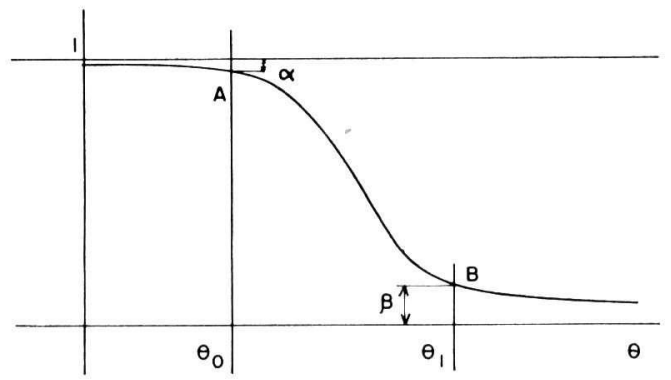

Fig. 1. - Courbe OC

de même

$$
L\left(\theta_{0}\right)=\operatorname{Pr}\left\{\text { accepter } H_{0} \text { si } \theta=\theta_{0}\right\}=1-\alpha .
$$

$L(\theta)$ est donc asymptotique à $L(\theta)=1$ et $L(\theta)=0$ et passe par $A$ si l'échantillonnage est classique, par $A$ et par $B$ si l'échantillonnage est progressif.

- si l'échantillonnage est classique, le choix du test et le choix du nombre de tirages conditionnent la forme de la courbe et le point $B$;

- si l'échantillonnage est progressif, le choix du test déterminera la forme de la courbe et le nombre moyen de tirages.

Quand nous comparions deux tests classiques, pour un nombre de tirages donné, nous préférions le test le plus puissant ( $1-\beta$ maximum), celui qui minimisait le risque de deuxième espèce.

Ici, nous choisirons le test qui demande un nombre moyen de tirages minimum (comparaison des courbes OC).

Le test choisi est dérivé des études de Neymann et Pearson et est décrit ci-après.

\section{3. - Le test progressif utilisé : le SPRT}

En se plaçant dans le cas de variables continues (ce qui ne restreint pas la généralité mais facilite l'écriture), on considère les deux hypothèses suivantes:

$$
\left\{H_{0}: f(x)=f\left(x, \theta_{0}\right)\right\} \quad \text { et } \quad\left\{H_{1}: f(x)=f\left(x, \theta_{1}\right)\right\}
$$


(où $f(x)$ est la densité de probabilité); la probabilité d'obtenir au $n$-ième stade l'échantillon $E:\left\{x_{1}, x_{2}, \ldots x_{n}\right\}$ est, s'il y a indépendance des $x_{i}$ :

a) si l'hypothèse $H_{0}$ est vraie, $P_{0 m}=f\left(x_{1}, \theta_{0}\right) f\left(x_{2}, \theta_{0}\right) \ldots f\left(x_{m}, \theta_{0}\right)$,

b) si l'hypothèse $H_{1}$ est vraie, $P_{1 m}=f\left(x_{1}, \theta_{1}\right) f\left(x_{2}, \theta_{1}\right) \ldots f\left(x_{m}, \theta_{1}\right)$.

Le (( sequential probability ratio test) (SPRT) consiste à adopter les critères de décisions suivants :

a) si $B<\frac{P_{1 m}}{P_{0 m}}<A$, prendre une nouvelle observation

b) $\begin{aligned} & \text { si } \frac{P_{1 m}}{P_{0 m}} \geqslant A \quad \text { accepter } H_{1} \\ & \text {. }\end{aligned}$

c) si $\frac{P_{1 m}}{P_{0 m}} \leqslant B \quad$ accepter $H_{0}$

$A$ et $B$ sont tels que les risques encourus soient $\alpha$ et $\beta$ : les quantités $A, B, \alpha, \beta$ sont liées par les relations suivantes :

$$
\left\{\begin{array}{l}
A \leqslant \frac{1-\beta}{\alpha} \\
B \geqslant \frac{\beta}{1-\alpha}
\end{array}\right.
$$

$A$ et $B$ étant difficiles à calculer, on prend pour simplifier très souvent

$$
A=\frac{1-\beta}{\alpha}, \quad B=\frac{\beta}{1-\alpha}
$$

et les risques réels encourus $\alpha^{\prime}$ et $\beta^{\prime}$ sont tels que

$$
\alpha^{\prime}+\beta^{\prime} \leqslant \alpha+\beta \text {. }
$$

\section{4. - Propriétés du SPRT}

\subsection{Efficacité}

Par rapport au test classique, le test progressif apporte une réduction dans le nombre d'observations à prendre en compte de $40 \%$ à peu près dans le cas du test de la moyenne d'une variable normale (WALD, 1947).

\subsection{Troncature}

Bien que la probabilité de prendre à la fin une des deux décisions $H_{0}$ ou $H_{1}$ soit un, par exemple, si la valeur réelle du paramètre se trouve entre les deux valeurs à tester, le nombre de tirages peut parfois être grand (voir 5.1.2).

Sans ( beaucoup ) changer les risques $\alpha, \beta$ (1) on peut décider qu'au stade $n_{0}$

(1) WaLD indique pour $\alpha=0,05, \beta=0.05$ que si

$n_{0}=1000, \alpha$ réel $<0.095, \beta$ réel $<0.095$

$n_{0}=2000, \alpha$ réel $<0.058, \beta$ réel $<0.058$

donc il faut prendre $n_{\mathrm{c}}$ grand, tres grand même si on considère ce qu'on prend généralement en expérimentation forestière 
on prendra la décision suivante pour arrêter définitivement l'expérience :

a) si $P_{1 m}>P_{0 m}$ accepter $H_{1}$,

b) si $P_{1 m}<P_{0 m}$ accepter $H_{0}$.

\subsection{Caractère approché du test}

La théorie du SPRT n'est qu'approchée en certains points et son application justifie d'autres simplifications, cela est parfois grave : si par exemple $\alpha$ et $\beta$ sont très différents (ils correspondent à des hypothèses aux conséquences très différentes), alors l'inégalité $\alpha^{\prime}+\beta^{\prime} \leqslant \alpha+\beta$ n'est plus satisfaisante étant donné qu'elle ne garantit pas $\left\{\begin{array}{l}\alpha^{\prime} \leqslant \alpha \\ \beta^{\prime} \leqslant \beta\end{array}\right.$ et on risque des échecs graves.

\subsection{Test valable dans le cas d'hypothèses simples seulement}

Le point le plus important du SPRT est que, du fait de sa nature, il ne peut servir que pour des hypothèses simples.

On a en effet écrit l'expression de probabilités de l'échantillon observé sous des hypothèses simples et la dérivation des bornes $A$ et $B$ à partir de $\alpha$ et $\beta$ ne peut être faite que dans le cas d'hypothèses simples.

WALD (1947) espérait que ce test était une procédure progressive optimale mais il n'a jamais réussi à la démontrer. On verra au paragraphe 5.12 des modifications. Il a été appliqué surtout à des distributions discrètes (binomiale, Poisson) et à quelques cas de distributions continues (normale, exponentielle - car elles conduisent à des résultats simples) (voir la bibliographie dans JoHNSON, 1961).

\section{3. - APPLICATIONS A LA LOI BINOMIALE}

\section{1. - Définition du problème}

Soit une variable binomiale $x$ prenant les valeurs 1 avec la probabilité $p$ et 0 avec la probabilité $1-p$.

On veut en général savoir si la moyenne $p$ (ou proportion de $x=1$ dans la population) est supérieure ou non à une valeur fixée au départ $p^{\prime}$.

$p^{\prime}$ représentera par exemple dans les contrôles de fabrication la proportion de rebuts tolérable à la vente (par exemple proportion déterminée par des analyses de comportement du client).

Nous avons vu l'impuissance de l'analyse séquentielle à traiter des hypothèses composées telles que $\left\{H^{\prime}: p>p^{\prime}\right\}$ ou $\left\{H: p \leqslant p^{\prime}\right\}$.

Il est possible de tourner cette difficulté :

a) on remarque d'abord que la probabilité de rejeter l'hypothèse $H$ quand $p>p^{\prime}$ est une fonction qui croît avec $p$, de même la probabilité d'accepter $H$ si $p \leqslant p^{\prime}$ croît quand $p$ décroît; 
$b$ ) ensuite on choisit $p_{0} \leqslant p^{\prime}$ (et $p_{1}>p^{\prime}$ ) de telle façon que, quand $p \leqslant p_{0}$ (et $p \geqslant p_{1}$ ) le rejet de $H$ (et l'acceptation de $H$ ) revêt une importance pratique (économique par exemple).

c) considérons les deux hypothèses :

$$
\begin{aligned}
& \left\{H_{0}: p=p_{0}\right\} \rightarrow \operatorname{Pr}\left\{\text { accepter } H_{1} \text { si } p=p_{0}\right\}=\alpha \\
& \left\{H_{1}: p=p_{1}\right\} \rightarrow \operatorname{Pr}\left\{\text { accepter } H_{0} \text { si } p=p_{1}\right\}=\beta
\end{aligned}
$$

Si nous prenons $p_{0}^{\prime}<p_{0}$ on a, d'après ce qui a été dit en $a$ ):

$$
\operatorname{Pr}\left\{\text { accepter } H_{1} \text { si } p=p_{0}^{\prime}\right\}<\operatorname{Pr}\left\{\text { accepter } H_{1} \text { si } p=p_{0}\right\}
$$

Tester les deux hypothèses $H_{0}$ et $H_{1}$ équivaut donc à tester les deux hypothèses $H_{0}^{\prime}$ et $H_{1}^{\prime}$

$\left\{H_{0}^{\prime}: p \leqslant p_{0}\right\}$ et $\left\{H_{1}^{\prime}: p \geqslant p_{1}\right\}$ qui sont les deux hypothèses exposées en $b$ ), donc à tester $\left\{H: p \leqslant p^{\prime}\right\}$.

\section{2. - Application du SPRT: exemple théorique}

Nous montrons sur cet exemple les possibilités de calcul du SPRT. Soit le $m$-ième stade d'un échantillonnage progressif et l'échantillon $E:\left\{x_{1}, x_{2} \ldots x_{m}\right\}$, la probabilité d'obtenir cet échantillon est simplement d'après les propriétés de la loi binomiale

$$
\pi=p^{\mathrm{d} m}(1-p)^{n-\mathrm{d} m}
$$

s'il y a $\mathrm{d}_{m}$ tirages où on a obtenu $x=1$.

Si $H_{0}$ est vrai, alors cette probabilité est $P_{0 m}=P_{0}^{\mathrm{d} m}\left(1-p_{0}\right)^{m-\mathrm{d} m}$.

Si $H_{1}$ est vrai, alors cette probabilité est $P_{1 m}=P_{1}^{\mathrm{d} m}\left(1-p_{1}\right)^{m-\mathrm{d} m}$.

On applique à ce moment le SPRT en passant aux logarithmes :

$$
\lg \frac{P_{1 m}}{P_{0 m}}=\mathrm{d}_{m} \lg \frac{P_{1}}{P_{0}}+\left(m-\mathrm{d}_{m}\right) \lg \frac{1-P_{1}}{1-P_{0}}
$$

et la règle de décision est $\left(\right.$ avec $\left.A=\frac{\beta}{1-\alpha}, B=\frac{\alpha}{1-\beta}\right)$

$$
\begin{array}{ll}
\lg A<\lg \frac{P_{1 m}}{P_{0 m}}<\lg B & \rightarrow \text { continuer } \\
\lg \frac{P_{1 m}}{P_{0 m}} \geqslant \lg B & \rightarrow \text { accepter } H_{1} \\
\lg \frac{P_{1 m}}{P_{0 m}} \leqslant \lg A & \rightarrow \text { accepter } H_{0}
\end{array}
$$




\section{3. - Applications de la technique}

C'est à la loi binomiale que l'analyse progressive a été la plus appliquée dans les contrôles de fabrication; dans le cas de produits présentant de multiples caractéristiques, des grilles déterminent si un individu doit être rebuté ou être bon et dans l'impossibilité de contrôler chaque individu, on contrôle des unités de fabrication sur leur proportion de rebuts.

Ce problème s'adapte fort bien aux livraisons de plants forestiers.

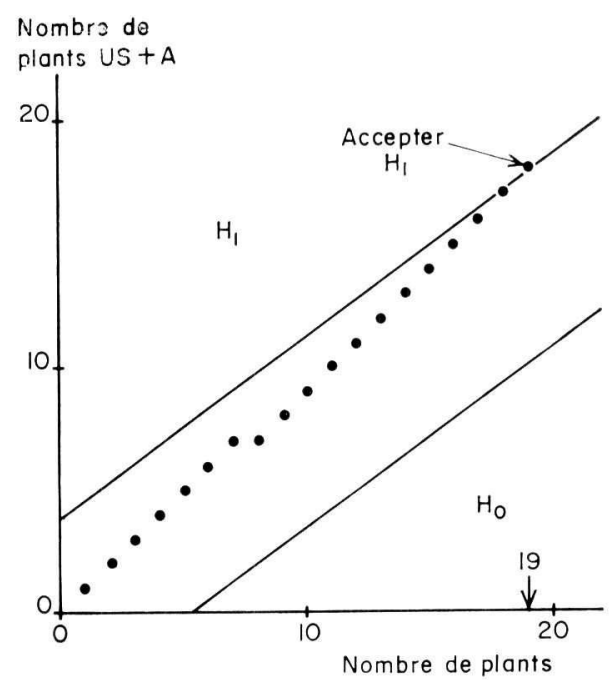

FIG. 2. - Echantillonnage progressif : application à la loi binomiale

En étudiant une provenance particulière (LA-FA) de sapin pectiné (AMELIO 67-039), on s'est donné les risques: $\alpha=\beta=0,05$ et les hypothèses afférentes.

$$
\left\{\begin{array}{l}
H_{0}: p \leqslant 0,65 \\
H_{1}: p \geqslant 0,80
\end{array} \text { où } p \text { est la proportion des plants (US }+\mathrm{A}\right. \text { ) }
$$

on a observé après tirage au hasard dans l'ordre

$$
7(\mathrm{US}+\mathrm{A}), 1 \mathrm{~B}, 12(\mathrm{US}+\mathrm{A}), 1 \mathrm{~B}, \ldots
$$

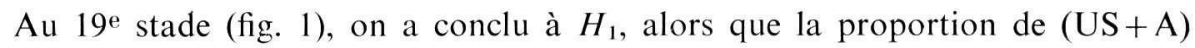
observée était $18 / 19=0,947$.

La table de Biometrika révèle que, dans le cas d'un échantillon de taille 19, $p=0,947$ n'est pas significativement différent de $p_{1}=0,80$, donc confirme les avantages de l'échantillonnage progressif: il aurait fallu 30 observations pour arriver à séparer $p$ de $p_{1}$ au seuil de $5 \%$ dans un test classique.

L'application de cette technique dans le cas de contrôle de l'état pathologique 
d'une forêt, de contrôle d'aménagements ou d'étude des possibilités de trouver dans une forêt des bois particuliers (bois de tranchage, etc.) reste à faire, (JEFFERS (1954)).

\section{4. - APPLICATIONS A LA LOI NORMALE}

\section{1. - Définition du problème}

Une distribution normale est uniquement caractérisée par deux paramètres, la moyenne $\mu$, la variance $\sigma^{2}$.

Un test statistique classique (taille $n$ déterminée) fournit à la fois une estimation de la moyenne $\mu$ et de la variance $\sigma^{2}$, et des moyens de vérifier des hypothèses telles que $\mu=\mu_{0}$ ou $\sigma^{2}=\sigma_{0}^{2}$.

L'analyse progressive permet seulement de tester des hypothèses simples : si on connaît donc la variance $\sigma^{2}$, deux problèmes peuvent se poser :

a) $\left\{H: \mu=\mu_{0}\right\}$ : on se donne $\delta$ tel qu'un écart de $\delta$ par rapport à $\mu$ soit d'une importance pratique et le problème revient à tester :

$$
\left\{H_{1}: \mu=\mu_{0}\right\} \text { contre }\left\{H_{2}: \mu=\mu_{0} \pm \delta\right\} .
$$

b) $\quad\left\{H: \mu=\mu_{0}\right\}$ contre $\left\{H^{\prime}: \mu=\mu_{1}\right\}$

dans la pratique industrielle, le problème $a$ ) est fréquent et consiste à vérifier le calibrage d'une fabrication. Il semble que dans les sciences agronomiques, le problème $b$ ) soit le plus fréquent.

\section{2. - Application du SPRT au deuxième cas}

On peut démontrer aussi facilement que dans le paragraphe 3.2 que les frontières entre les zones d'acceptation d'une hypothèse et celle d'indécision sont des droites dans le cas $b$ ).

Disposant donc d'une évaluation a priori de $\sigma^{2}$ (expériences préalables, etc.) de $\mu_{0}, \mu_{1}, \alpha, \beta$, on peut démontrer que les droites ont pour équation :

$$
\begin{aligned}
& y_{1}=\frac{\sigma^{2}}{\mu_{1}-\mu_{0}} \lg \frac{\beta}{1-\alpha}+m \frac{\mu_{0}+\mu_{1}}{2} \\
& y_{2}=\frac{\sigma^{2}}{\mu_{1}-\mu_{0}} \lg \frac{1-\beta}{\alpha}+m \frac{\mu_{0}+\mu_{1}}{2}
\end{aligned}
$$

$m$ : taille de l'échantillon, et on porte en ordonnées la somme des valeurs observées $\Sigma x$; on adopte la règle de décision suivante :

$$
\begin{cases}\Sigma x>y_{1} & : \text { accepter } H: \mu=\mu_{0} \\ y_{1} \geqslant \Sigma x \geqslant y_{2} & : \text { continuer 1'échantillonnage } \\ \Sigma x<y_{2} & : \text { accepter } H^{\prime}: \mu=\mu_{1}\end{cases}
$$




\section{3. - Exemples pratiques}

JEFFERS (1954), donne un exemple d'application à l'entomologie forestière et des possibilités d'application à la sylviculture. On peut, en appliquant cette technique, juger si une forêt possède un nombre de réserves suffisant pour pouvoir justifier un traitement feuillu ou sinon entraîner l'enrésinement.

IvEs (1954), et STARK (1952), en particulier, (voir 6.2), ont traité des applications à l'entomologie : en étudiant la distribution des œufs d'insectes sur les feuilles, après avoir vérifié qu'elle est normale de variance connue (études préalables), après avoir déterminé trois niveaux:
a) $\mu<\mu_{0}$
: la forêt est saine
b) $\mu_{1}<\mu<\mu_{2}$ : la forêt est justiciable d'une intervention
c) $\mu>\mu_{3} \quad$ : la forêt de toute façon mourra

on voit dans quelle catégorie ranger la forêt étudiée.

Exemple: la hauteur des plants de sapin pectiné au sortir de la pépinière (Amelio. 67-039) est supposée normale, d'écart-type 3,16 cm.

On tire au hasard dans la provenance LA-FA pour savoir si :

$$
\left\{\begin{array}{llll}
1 & \left\{H_{1}: h>19,5\right\} & \text { ou } & \left\{H_{1}^{\prime}: h<18,5\right\} \\
2 & \left\{H_{2}: h>20\right\} & \text { ou } & \left\{H_{2}^{\prime}: h<18\right\} \\
3 & \left\{H_{3}: h>21\right\} & \text { ou } & \left\{H_{3}^{\prime}: h<19\right\} \\
4 & \left\{H_{4}: h>20,5\right\} & \text { ou } & \left\{H_{4}^{\prime}: h<19,5\right\}
\end{array}\right.
$$

Ces quatre couples ont été choisis alors que la vraie moyenne est 20,08 (calculée sur 180 individus) (tableau 1).

\section{TABLEAU 1}

Hauteurs des plants avec leur numéro de tirage

\begin{tabular}{|c|c|c|c|c|c|c|c|c|c|c|c|c|c|}
\hline Numéro du tirage $\ldots \ldots \ldots$ & 1 & 2 & 3 & 4 & 5 & 6 & 7 & 8 & 9 & 10 & 11 & 12 & 13 \\
\hline Hauteur .............. & 16 & 24 & 22 & 20 & 22 & 20 & 26 & 22 & 16 & 16 & 20 & 18 & 24 \\
\hline Numéro du tirage $\ldots . .$. & 14 & 15 & 16 & 17 & 18 & 19 & 20 & 21 & 22 & 23 & 24 & 25 & 26 \\
\hline Hauteur & 22 & 16 & 24 & 26 & 18 & 20 & 22 & 18 & 20 & 20 & 18 & 24 & 24 \\
\hline
\end{tabular}

Les frontières entre les zones d'indécision et d'acceptation d'hypothèses sont $\left(F\left(I, H_{1}\right)\right.$ est l'équation de la droite frontière entre $I$ l'indifférence de $H_{1}$ l'hypothèse considérée) : 


$$
\begin{array}{ll}
1 y=-29,44+19 m\left(F\left(I, H_{1}^{\prime}\right)\right), \quad z=29,44+19 m\left(F\left(I, H_{1}\right)\right) \\
2 y=-14,72+19 m\left(F\left(I, H_{2}^{\prime}\right)\right), \quad z=14,72+19 m\left(F\left(I, H_{2}\right)\right) \\
3 y=-14,72+20 m\left(F\left(I, H_{3}^{\prime}\right)\right), \quad z=14,72+20 m\left(F\left(I, H_{3}\right)\right) \\
4 y=-29,44+20 m\left(F\left(I, H_{4}^{\prime}\right)\right), \quad z=29,44+20 m\left(F\left(I, H_{4}\right)\right)
\end{array}
$$

Sur cet exemple simple, on a l'illustration des propriétés suivantes (fig. 3 et 4) :

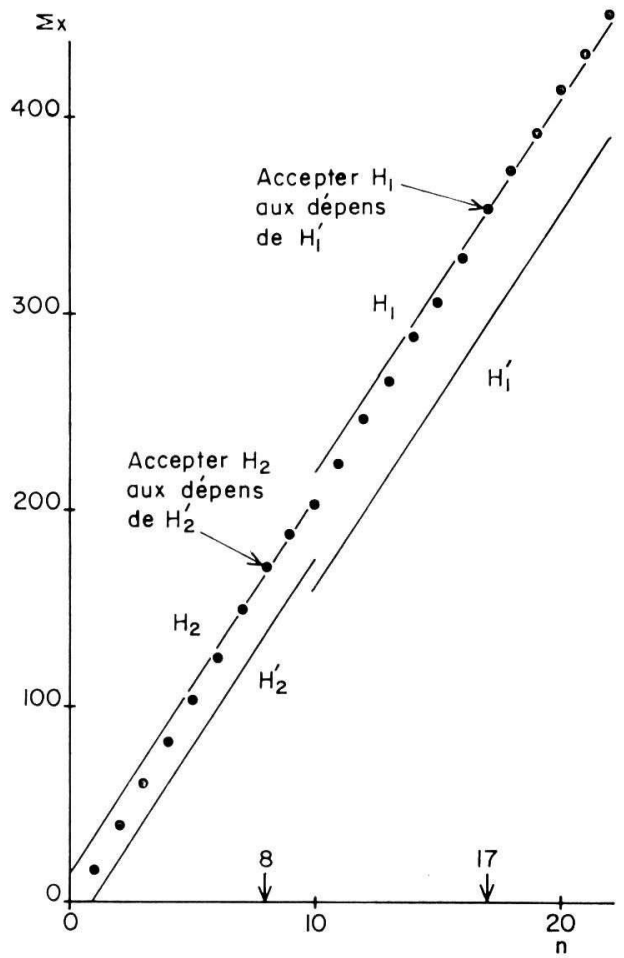

FIG. 3. - Echantillonnage progressif : applications à la loi normale (Hypothèses 1 et 2)

- plus l'écart est grand entre les deux hypothèses à tester, moins il faut tirer d'individus ;

- si la valeur réelle du paramètre est entre les deux valeurs à tester, le nombre de tirages est grand. En testant $H_{4}$ contre $H_{4}^{\prime}, H_{4}$ est accepté au $55^{\mathrm{e}}$ tirage (paragraphe 5.12);

- si $\sigma^{2}$ est sous-estimé, on crée de gros risques non contrôlables; si $\sigma^{2}=12$, $H_{2}$ n'est accepté par exemple qu'au $15^{\mathrm{e}}$ tirage (voir paragraphe 5.11 , les possibilités du (( test $t$ progressif )) : on a donc intérêt à surestimer légèrement les paramètres qu'on ne teste pas. 


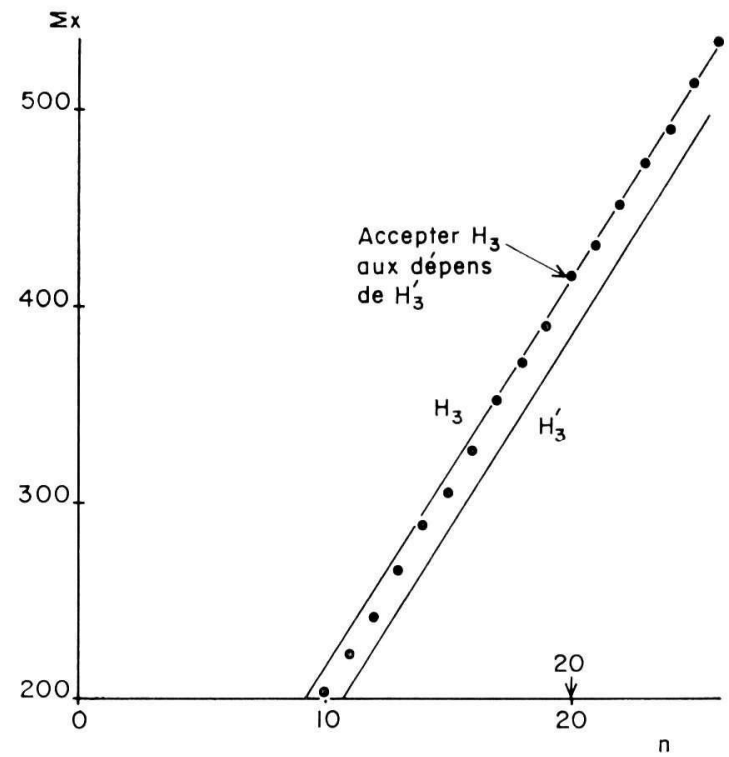

FIG. 4. - Echantillonnage progressif : application à la loi normale hypothèses 3 (les points sont les mêmes que sur la figure 3)

\section{5. - CONCLUSION}

\section{1. - Les défauts de l'analyse progressive}

\subsection{Nature des hypothèses}

Lorsqu'il n'y a qu'un paramètre à déterminer, il est relativement facile de résoudre le problème des hypothèses composées; au prix d'hypothèses simples mais clarificatrices, on peut tourner la difficulté. Mais quand deux paramètres varient, cela devient très difficile. Ainsi WALD a-t-il quand même réussi à développer un test $t$ progressif (où la moyenne et la variance d'une population normale sont inconnues) (1). Mais, d'après JoHNSON, cette méthode est loin d'être satisfaisante.

\subsection{Rapidité d'obtention du résultat}

Quand la valeur réelle du paramètre dans la population $\mu$ tombe entre les deux valeurs $\mu_{0}$ et $\mu_{1}$ qu'on veut tester, il est évident que la procédure progressive perd de son efficacité et on a souvent recours à la possibilité de tronquer la procédure.

(1) WALD transforme son hypothèse composée en hypothèse simple de la façon suivante : en admettant a priori une distribution pour les paramètres qui varient dans l'hypothèse composée, il pondère de cette façon les différentes hypothèses élémentaires, et en faisant une sommation obtient une hypothèse simple. 
Si vraiment le choix entre les deux hypothèses est lourd de conséquences, la troncature indépendamment du fait qu'elle donne un nombre $n_{0}$ relativement important de tirages, est une procédure discutable.

$\mathrm{Si}$ on n'adopte pas la troncature, le gain procuré par le SPRT comparé aux tests de taille fixe est ridiculement faible : avec $\alpha=0,01$ et $\mu=\left(\mu_{0}+\mu_{1}\right) / 2$, le gain est de 528 contre 541 tirages. L'avantage de l'échantillon progressif est nul.

ANDERSON (1960) a amélioré le SPRT dans ce cas là. Le gain est considérable (dans le même cas 402 contre 541 tirages) mais le mode opératoire est sensiblement compliqué.

\subsection{Dangers de l'échantillonnage}

L'échantillonnage simple au hasard classique pêche par un aspect particulier :

- il ne tient pas compte de l'hétérogénéité de la population : donc la variance de l'estimation des paramètres sera grande;

— si nous prenons un petit échantillon, il n'est pas sûr qu'il représentera les hétérogénéités de la population.

Le premier remède était l'échantillonnage stratifié basé sur l'analyse de variance et qui, en séparant des sous-populations homogènes en leur intérieur mais hétérogènes entre elles, diminuait la variance d'erreur, cela dans le cas où les souspopulations étaient facilement repérables.

Le second remède était l'échantillonnage systématique; dans le cas où on ne pouvait pas repérer les sous-populations, on essayait au moins d'obtenir une représentation valable de l'ensemble de notre population par la systématisation du sondage : cela était particulièrement vrai dans l'échantillonnage de superficies (forestières par exemple).

On ne savait pas très bien calculer la variance mais on connaissait en général une borne supérieure de cette variance et on était sûr de ne pas avoir commis de fautes (1).

Il est évident que l'échantillonnage progressif actuel étant issu de l'échantillonnage simple au hasard, il n'est pas possible d'utiliser les possibilités de la stratification, et que procéder à un tirage systématique progressif consiste à nier au départ les raisons pour lesquelles on adopte l'échantillonnage systématique.

\subsection{Difficulté des calculs}

Les calculs, même dans les cas simples, sont compliqués, et il importe que les biométriciens puissent fournir des abaques d'emploi simple pour faciliter la tâche des expérimentateurs.

C'est d'ailleurs à ce prix que l'échantillonnage progressif pourra être payant.

(1) Voir dans «Sampling Techniques» (COCHRAN et Cox, Wiley) les rapports entre échantillonnage stratifié et échantillonnage systématique. De plus, l'échantillonnage systématique est plus facile à planifier et à réaliser. 


\subsection{Analyse multivariable}

La somme de travail théorique, dévolue à l'échantillonnage progressif sur des individus sur lesquels on mesure plusieurs caractères, est quasiment nulle.

\subsection{Estimation des paramètres}

L'analyse progressive étant surtout un outil de décision, l'estimation d'un paramètre en vue d'études futures et de comparaison est une question accessoire.

\section{2. - Les avantages}

\subsection{Le gain}

Malgré certaines limites exposées en 5.12, l'analyse progressive permet d'épargner un nombre considérable de tirages (voir partie 2.3). Dans le cas où les caractères sont de mesure difficile, le gain peut être énorme (en coût).

\subsection{Une autre façon de raisonner statistiquement}

Un autre avantage de cette technique est qu'elle nécessite une étude très serrée de ce que l'on veut attendre d'une expérience.

Une expérience classique permet à un praticien prudent de se réfugier dans un (( wait and see ) souvent infécond, parfois désabusé.

L'analyse progressive exige une définition stricte des hypothèses de départ, une évaluation (( économique)) des risques à prendre : par exemple, dans l'expérimentation d'un médicament, on ne donnera pas de risques équivalents à l'hypothèse $\{H$ : bon médicament $\}$ et à $\left\{H^{\prime}\right.$ : mauvais médicament $\}$. Elle oblige en quelque sorte à abandonner la neutralité et à adopter une position dynamique (ce qui ne veut pas dire non objective).

\subsection{Répétition d'expérience}

Du fait que l'on a fixé au départ les deux risques $\alpha$ et $\beta$, on peut être assuré que dans une longue série d'expériences similaires, l'acceptation d'une hypothèse aux dépens d'une autre sera une mauvaise décision dans $100 \beta$ cas sur 100 et qu'on aura eu tort de refuser l'hypothèse dans $100 \alpha \%$ des cas.

Dans un échantillonnage classique, du fait que $\beta$ est déterminé a posteriori, et d'ailleurs n'est à ce moment jamais calculé, on ne sait pas chiffrer cette probabilité de faire un mauvais choix en acceptant $H$.

L'analyse progressive permet d'être certain des risques qu'on encourt.

\section{3. - L'avenir de la méthode}

La liste des péchés est fort longue et malgré l'importance que nous accordons à la section 5.2, on pourrait penser que dans l'état actuel, l'analyse progressive 
est seulement un puissant moyen de contrôle ou un exercice d'école statistique mais pas un outil fréquemment utilisé dans la recherche.

Néanmoins, le bouillonnement théorique est tel qu'on peut espérer des développements rapides dans certaines directions.

\subsection{Démonstration d'Anscombe}

ANSCOMBE a montré que, pourvu que le nombre de tirages $n$ soit assez grand, les méthodes employées pour l'estimation des paramètres dans le cas classique ( $n$ fixe) étaient également valables pour l'échantillonnage progressif.

La restriction est bien évidente : quand $n$ est petit, la précision des estimateurs laisse beaucoup à désirer (que l'échantillonnage soit fixe ou progressif).

\subsection{Intervalles de confiance de longueur fixée}

L'échantillonnage classique ne donne pas la possibilité de calculer des intervalles de confiance de longueur fixée; un type spécial d'échantillonnage progressif résolvant le problème est le suivant : un premier stade est l'estimation de la variance dans un échantillon de taille $n_{0}$ puis on détermine $n_{0}+n$ tel que la longueur de l'intervalle de confiance soit égal à la longueur fixée et on tire les $n$ individus supplémentaires.

\subsection{Développement de l'analyse progressive multivariable}

On pourra traiter les problèmes d'analyse discriminante classique progressivement. L'application de la technique sera ici immédiate; un individu n'étant plus déterminé par une mesure mais par un ensemble de $p$ mesures, le coût de l'échantillonnage s'en trouve très sensiblement augmenté.

\subsection{Tests progressifs non paramétriques}

Un test non paramétrique est un test qui ne fait pas d'hypothèses sur la forme des distributions; leur efficacité (simplicité des calculs, perte d'information très faible dans certains cas) en fait des outils très précieux pour tester une hypothèse. Leur principe est de se ramener à la loi binomiale (ou multinomiale); du fait de la facilité du traitement de la loi binomiale en analyse progressive, ces tests ont des versions séquentielles et KendaLL (1961) affirme que leur efficacité est remarquable.

L'analyse progressive représente un état particulier de la statistique, une méthode de raisonnement neuve, un point de départ pour la théorie de la décision et un outil très efficace dans certaines conditions. Son application aux sciences forestières et agronomiques est encore limitée mais dans la mesure où le but de travail peut être chiffré, elle permet d'arriver à des plans d'expérimentation efficaces. Il est donc important d'avoir cette technique à l'esprit dans l'avenir. 


\section{SUMMARY}

\section{A STATISTICAL METHOD : SEQUENTIAL ANALYSIS}

Principles of sequential analysis are discussed including an introduction to the notion of power. The value of the method is shown in applications to forest research (binomial and normal case) and discussed in the last chapter. Although it is imperfect, it remains a valuable way of thinking (statistically) and building new experiments.

\section{ZUSAMMENFASSUNG}

\section{EINE STATISTISCHE METHODE : DIE SEQUENTIELLE ANALYSIS}

Grundlagen der sequentiellen Analysis sind mit einer Einleitung der Vorstellung von « power » darstellt. Der Vorteil der Methode erscheint in Waldforschung Anwendungen und ist dem letzten Teil besprochen. Trotz sciner Unvollkommenheiten ist die sequentielle Analysis ein neues statistisches Mittel um «gut » zu experimentieren.

\section{RÉFÉRENCES BIBLIOGRAPHIQUES}

\section{ASPECT THÉORIQUE}

ANDERSON T.W., 1960. A modification of the sequential probability ratio test to reduce the sample size. Ann. Math. Stat., 31, 165-197.

Chernoff H., 1959. Sequential design of experiments. Ann. Math. Stat., 30, 775-780.

JEFFERS J.N.R., 1954. The application of sequential sampling to forest problems. Statistic Section Paper $n^{\circ} 4$, Alice Holt.

Johnson N.L., 1961. Sequential analysis : a survey. J. r. Stat. Soc. Série A, 372-411.

Johnson N.L., Leone F.C., 1964. Statistics and experimental design, t. 2, 522 p., Wiley and Sons, New York.

Kendall M.G., Stuart A., 1961. The advanced theory of statistics t. 2, 676 p., Grifin, London.

SCHeffe H., 1959. The analysis of variance, $476 \mathrm{p}$. Wiley and Sons, New York.

TANG P.C., 1938. The power function of the analysis of variance tests with tables and illustration of their use. Stat. Res. Men., 2, 126.

WALD A., 1947. Sequential analysis, 212 p., Wiley and Sons, New York.

\section{ASPECT PRATIQUe}

Ives W.G.H., 1954. Sequential sampling of insect populations. Forestry Chronicle, 30 (3), 287-291.

Ives W.G.H., Warren G.L., 1965. Sequential sampling for white grubs, Canad. Entomol., 97 (6), 596-604.

Oakland G.B., 1951. Sequential sampling Biometrics Unit, Sci. Serv. Canad. Dept. Agric.

Stark R.W., 1952. Sequential sampling of the lodgepole needle miner. Forestry Chronicle, 28 (1), 57-60.

Stevens R.E., Stark R.W., 1962. Sequential sampling for the lodgepole needle miner. J. econ. Entomol., 55 (4), 491-494. 